\title{
Dupilumab-Induced Facial Flushing After Alcohol Consumption
}

\author{
Nicholas D. Brownstone, MD; Vidhatha Reddy, BA; Quinn Thibodeaux, MD; Bridget Myers, BS; \\ Stephanie Chan, BS; Wilson Liao, MD; Tina Bhutani, MD
}

\section{PRACTICE POINTS}

- Dupilumab is a fully humanized monoclonal antibody that inhibits the action of IL-4 and IL-13. It was approved by the US Food and Drug Administration in 2017 for treatment of moderate to severe atopic dermatitis.

- Facial flushing after alcohol consumption may be an emerging side effect of dupilumab.

- Whether dupilumab influences enzymes involved in processing alcohol requires further study.

Dupilumab is a biologic agent approved by the US Food and Drug Administration for the treatment of atopic dermatitis (AD). Here, we report 2 patients with $A D$ who were treated with dupilumab and subsequently developed facial flushing after consuming alcohol. A possible mechanism of action for this side effect is discussed along with a potential role of dupilumab.

Cutis. 2021;108:106-107.

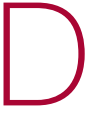
upilumab is a fully humanized monoclonal antibody to the $\alpha$ subunit of the IL- 4 receptor that inhibits the action of helper $\mathrm{T}$ cell $\left(\mathrm{T}_{\mathrm{H}} 2\right)$-type cytokines IL-4 and IL-13. Dupilumab was approved by the US Food and Drug Administration (FDA) in 2017 for the treatment of moderate to severe atopic dermatitis (AD). We report 2 patients with $\mathrm{AD}$ who were treated with dupilumab and subsequently developed facial flushing after consuming alcohol.

\section{Case Report}

Patient 1-A 24-year-old woman presented to the dermatology clinic with a lifelong history of moderate to severe AD. She had a medical history of asthma and seasonal allergies, which were treated with fexofenadine and an inhaler, as needed. The patient had an affected body surface area of approximately $70 \%$ and had achieved only partial relief with topical corticosteroids and topical calcineurin inhibitors.

Because her disease was severe, the patient was started on dupilumab at FDA-approved dosing for AD: a 600-mg subcutaneous (SC) loading dose, followed by $300 \mathrm{mg}$ SC every 2 weeks. She reported rapid skin clearance within 2 weeks of the start of treatment. Her course was complicated by mild head and neck dermatitis.

Seven months after starting treatment, the patient began to acutely experience erythema and warmth over the entire face that was triggered by drinking alcohol (Figure). Before starting dupilumab, she had consumed alcohol on
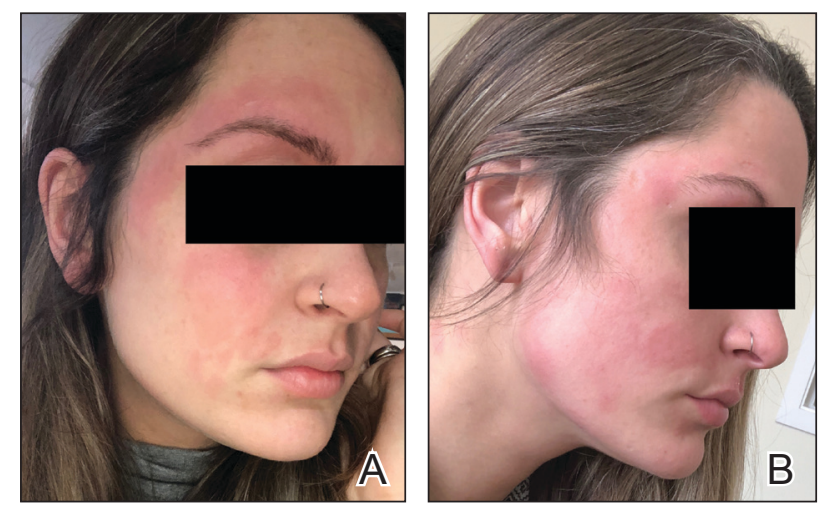

Acute erythema and warmth over the entire face triggered by alcohol consumption in a 24-year-old woman who had started treatment with dupilumab 7 months prior. A, Frontal facial view. B, Side facial view showing acute erythema concentrated around the eyebrows, cheeks, and jawline.

From the Department of Dermatology, Psoriasis and Skin Treatment Center, University of California San Francisco.

Drs. Brownstone and Thibodeaux, Mr. Reddy, Ms. Myers, and Ms. Chan report no conflict of interest. Dr. Liao has received research grant funding from AbbVie, Amgen, Janssen Pharmaceuticals, LEO Pharma, Novartis, Pfizer, Regeneron Pharmaceuticals, and TRex Bio. Dr. Bhutani has received research grants from Regeneron Pharmaceuticals.

Correspondence: Nicholas D. Brownstone, MD, Psoriasis and Skin Treatment Center, Department of Dermatology, University of California San Francisco, 515 Spruce St, San Francisco, CA 94118 (Nicholas.Brownstone@ucsf.edu). doi: $10.12788 /$ cutis.0316 
multiple occasions without a flushing effect. This new finding was distinguishable from her facial dermatitis. Onset was within a few minutes after drinking alcohol; flushing self-resolved in 15 to 30 minutes. Although diffuse, erythema and warmth were concentrated around the jawline, eyebrows, and ears and occurred every time the patient drank alcohol. Moreover, she reported that consumption of hard (ie, distilled) liquor, specifically tequila, caused a more severe presentation. She denied other symptoms associated with dupilumab.

Patient 2-A 32-year-old man presented to the dermatology clinic with a 10-year history of moderate to severe AD. He had a medical history of asthma (treated with albuterol, montelukast, and fluticasone); allergic rhinitis; and severe environmental allergies, including sensitivity to dust mites, dogs, trees, and grass.

For $\mathrm{AD}$, the patient had been treated with topical corticosteroids and the Goeckerman regimen (a combination of phototherapy and crude coal tar). He experienced only partial relief with topical corticosteroids; the Goeckerman regimen cleared his skin, but he had quick recurrence after approximately 1 month. Given his work schedule, the patient was unable to resume phototherapy.

Because of symptoms related to the patient's severe allergies, his allergist prescribed dupilumab: a 600-mg SC loading dose, followed by $300 \mathrm{mg}$ SC every 2 weeks. The patient reported near-complete resolution of $\mathrm{AD}$ symptoms approximately 2 months after initiating treatment. He reported a few episodes of mild conjunctivitis that selfresolved after the first month of treatment.

Three weeks after initiating dupilumab, the patient noticed new-onset facial flushing in response to consuming alcohol. He described flushing as sudden immediate redness and warmth concentrated around the forehead, eyes, and cheeks. He reported that flushing was worse with hard liquor than with beer. Flushing would slowly subside over approximately 30 minutes despite continued alcohol consumption.

\section{Comment}

Two other single-patient case reports have discussed similar findings of alcohol-induced flushing associated with dupilumab. ${ }^{1,2}$ Both of those patients-a 19-year-old woman and a 26-year-old woman-had not experienced flushing before beginning treatment with dupilumab for $\mathrm{AD}$. Both experienced onset of facial flushing months after beginning dupilumab even though both had consumed alcohol before starting dupilumab, similar to the cases presented here. One patient had a history of asthma; the other had a history of seasonal and environmental allergies.

Possible Mechanism of Action-Acute alcohol ingestion causes dermal vasodilation of the skin (ie, flushing). ${ }^{3}$ A proposed mechanism is that flushing results from direct action on central vascular-control mechanisms. This theory results from observations that individuals with quadriplegia lack notable ethanol-induced vasodilation, suggesting that ethanol has a central neural site of action. Although some research has indicated that ethanol might induce these effects by altering the action of certain hormones (eg, angiotensin, vasopressin, and catecholamines), the precise mechanism by which ethanol alters vascular function in humans remains unexplained. ${ }^{3}$

Deficiencies in alcohol dehydrogenase $(\mathrm{ADH})$, aldehyde dehydrogenase 2, and certain cytochrome P450 enzymes also might contribute to facial flushing. People of Asian, especially East Asian, descent often respond to an acute dose of ethanol with symptoms of facial flushingpredominantly the result of an elevated blood level of acetaldehyde caused by an inherited deficiency of aldehyde dehydrogenase $2{ }^{4}$ which is downstream from $\mathrm{ADH}$ in the metabolic pathway of alcohol. The major enzyme system responsible for metabolism of ethanol is ADH; however, the cytochrome P450-dependent ethanol-oxidizing system-including major CYP450 isoforms CYP3A, CYP2C19, CYP2C9, CYP1A2, and CYP2D6, as well as minor CYP450 isoforms, such as CYP2E1 - also are involved, to a lesser extent. ${ }^{5}$

A Role for Dupilumab? - A recent pharmacokinetic study found that dupilumab appears to have little effect on the activity of the major CYP450 isoforms. However, the drug's effect on ADH and minor CYP450 minor isoforms is unknown. Prior drug-drug interaction studies have shown that certain cytokines and cytokine modulators can markedly influence the expression, stability, and activity of specific CYP450 enzymes. ${ }^{6}$ For example, IL-6 causes a reduction in messenger RNA for CYP3A4 and, to a lesser extent, for other isoforms. ${ }^{7}$ Whether dupilumab influences enzymes involved in processing alcohol requires further study.

\section{Conclusion}

We describe 2 cases of dupilumab-induced facial flushing after alcohol consumption. The mechanism of this dupilumab-associated flushing is unknown and requires further research.

\section{REFERENCES}

1. Herz S, Petri M, Sondermann W. New alcohol flushing in a patient with atopic dermatitis under therapy with dupilumab. Dermatol Ther. 2019;32:e12762. doi:10.1111/dth.12762

2. Igelman SJ, Na C, Simpson EL. Alcohol-induced facial flushing in a patient with atopic dermatitis treated with dupilumab. JAAD Case Rep. 2020;6:139-140. doi:10.1016/j.jdcr.2019.12.002

3. Malpas SC, Robinson BJ, Maling TJ. Mechanism of ethanol-induced vasodilation. J Appl Physiol (1985). 1990;68:731-734. doi:10.1152/ jappl.1990.68.2.731

4. Brooks PJ, Enoch M-A, Goldman D, et al. The alcohol flushing response: an unrecognized risk factor for esophageal cancer from alcohol consumption. PLoS Med. 2009;6:e50. doi:10.1371/journal.pmed.1000050

5. Cederbaum AI. Alcohol metabolism. Clin Liver Dis. 2012;16:667-685. doi:10.1016/j.cld.2012.08.002

6. Davis JD, Bansal A, Hassman D, et al. Evaluation of potential disease-mediated drug-drug interaction in patients with moderateto-severe atopic dermatitis receiving dupilumab. Clin Pharmacol Ther. 2018;104:1146-1154. doi:10.1002/cpt.1058

7. Mimura H, Kobayashi K, Xu L, et al. Effects of cytokines on CYP3A4 expression and reversal of the effects by anti-cytokine agents in the three-dimensionally cultured human hepatoma cell line FLC-4. Drug Metab Pharmacokinet. 2015;30:105-110. doi:10.1016/j.dmpk.2014.09.004 